SUSX-TH-95/74

hep-ph/9510434

October 1995

\title{
Cosmic Electroweak Strings
}

\author{
Michael Goodband* and Mark Hindmarsh ${ }^{\dagger}$ \\ School of Mathematical and Physical Sciences \\ University of Sussex \\ Brighton BN1 9QH \\ $U K$
}

\begin{abstract}
We examine the Standard Model field configurations near cosmic strings in a particular class of models. This class is defined by the condition that the generator of the flux in the string, $T_{s}$, commutes with the Standard Model Lie algebra. We find that if the Standard Model Higgs carries a charge $F_{h} / 2$ under $T_{s}$, cosmic string solutions have Z-flux $\Phi_{Z}=\left[n-F_{h} N / F_{\phi}\right] 4 \pi \cos \theta_{w} / g$, where $n$ is any integer and $4 \pi N / q F_{\phi}$ is the flux of the gauge field associated with $T_{s}$. Only the configuration with the smallest value of $\left|n-F_{h} N / F_{\phi}\right|$ is stable, however. We argue that the instabilities found at higher $\Phi_{Z}$ are just associated with paths in configuration space reducing $\left|n-F_{h} N / F_{\phi}\right|$ by one unit. This contradicts recent claims that the instabilities in such models represent the spontaneous generation of current along the string. We also show that the stable strings have no Standard Model fermion zero modes: therefore there is no possibility of supercurrents carried by Standard Model particles in this class of models.
\end{abstract}

*e-mail: m.j.goodband@sussex.ac.uk

${ }^{\dagger}$ e-mail: m.b.hindmarsh@sussex.ac.uk 
In Grand Unified Theories (GUT) of particle physics with spontaneous symmetry breaking (SSB) there are often topological defects [1, 2]. In fact if the symmetries of nature are unified into one simple Lie group $G$, then there must exist topological monopoles. The mass density from such monopoles in a cosmological setting would dominate the universe, and this is not observed. This is the monopole problem, for which a number of solutions have been proposed; the leading contenders being inflation or the formation of strings at a later SSB which connect monopole and anti-monopole and lead to their annihilation.

The existence of other topological defects, specifically domain walls and strings, are dependent on the details of the SSBs present in the model. If domain walls are formed they too will dominate the mass density of the universe. This can be avoided by inflation or strings carving up the walls. Such considerations of cosmological implications can lead to restrictions on the allowable GUTs [3].

Topological strings on the other hand generally do not lead to cosmological catastrophies. In fact strings are considered as a possible source of large scale structure in the universe.

Witten showed [4], that for some particle physics models strings could be superconducting and support very large currents, via two different mechanisms. The first involves the occurrence of a charged scalar condensate, the other involves the appearance of fermion zero modes on the string. A third mechanism using charged vector bosons was later identified [5, 6]. All three types depend upon the details of the GUT model and none are generic.

Recent papers [7, 8], however, have argued that all GUT scale strings become superconducting at the electroweak symmetry breaking, and furthermore that a supercurrent spontaneously develops without an applied electric field. This could have serious cosmological implications, not least because superconducting string loops can shrink to form stable rings, and a population of such loops are as disastrous as topological monopoles.

It is therefore important to check that GUT strings are 'generically' superconducting, and in doing so we return to an old question: how does a cosmic string affect the fields of the electroweak theory in its vicinity?

So consider a GUT with two symmetry breakings and a lagrangian of the form

$$
\mathcal{L}=-\frac{1}{4} G_{\mu \nu}^{a} G^{\mu \nu a}+\operatorname{tr}\left[\left(D_{\mu} \phi\right)^{\dagger}\left(D^{\mu} \phi\right)\right]+\operatorname{tr}\left[\left(D_{\mu} H\right)^{\dagger}\left(D^{\mu} H\right)\right]+V(\phi, H)
$$

where $D_{\mu}$ is the covariant derivative, and $V(\phi, H)$ is some general gauge invariant fourth order potential. Now $\phi$ will acquire a vacuum expectation value (vev), which we take to be at the GUT scale of $10^{16} \mathrm{GeV}$, and breaks the symmetry group $G$ down to $G_{1}$, then $H$ acquires a vev at a lower energy scale and breaks the symmetry group $G_{1}$ down further to $G_{2}$. In general there can be more stages of symmetry breaking, but we are only considering two for simplicity. At the first symmetry breaking some of the various components of the scalar field $H$ will acquire masses, while a subset of the $H$ fields will develop an effective potential that will lead to the second SSB. We will take this subset 
to be such that its elements can be identified with the Higgs doublet of the electroweak standard model, and so the second symmetry breaking occurs at the electroweak scale of $10^{2} \mathrm{GeV}$.

Now suppose that the first homotopy group for the first symmetry breaking is nontrivial, $\pi_{1}\left(G / G_{1}\right) \neq 0$, so that there are stable string solutions with the asymptotic forms

$$
\phi=\phi_{0} \exp \left(i T_{s} \theta\right), \quad X_{\theta}^{s}=T_{s} / g r
$$

as $r \rightarrow \infty$, where $T_{s}$ is the string generator.

Now consider the terms that may be present in the lagrangian above that couple the GUT string fields to the Standard Model fields. There may be a cross term in the potential $|\phi|^{2}|h|^{2}$ between the GUT scalar $\phi$ and the Higgs doublet $h$, and the Standard Model covariant derivative may contain an additional term proportional to $X_{\mu}^{s} h$. These terms were stated in ref. [8] to be the most general terms coupling the GUT string fields to the Standard Model fields. However, there are a number of other terms that may be present in the lagrangian above. For example, there could be terms of the form $\operatorname{tr}\left(\left(\partial_{\mu} \mathcal{H}\right) X^{s \mu} h\right)$ and $\operatorname{tr}\left(\mathcal{H} X_{\mu} X^{s \mu} h\right)$ where $\mathcal{H}$ is a component of $H$ which is orthogonal to $h, X_{\mu}^{s}$ is the string gauge field and $X_{\mu}$ is some other vector boson. The GUT string may be unstable to solutions with non-zero values of the fields $\mathcal{H}, h$ and $X_{\mu}$ which could possibly give a charged condensate on the string. Whether this possibility is realised or not would depend upon the details of the GUT model.

So we eliminate these later sort of terms from consideration by assuming that the string generator commutes with all the electroweak generators i.e.

$$
\left[T_{s}, \tau^{a}\right]=0 \quad \text { and } \quad\left[T_{s}, Y\right]=0
$$

where $\tau^{a}$ are the weak isospin generators and $Y$ is the hypercharge generator. Note that this implies that the GUT string cannot be superconducting in the sense of Everrett, because $\left[T_{s}, Q\right]=0$ where $Q$ is the charge generator.

We will further assume that the GUT string is not superconducting in the sense of Witten either, because the effect of the electroweak phase transition on a superconducting string has already been considered in refs. [9, 10].

To illustrate the effect of the potential term and the addition of $X_{\mu}^{s} h$ to the covariant derivative, we will follow refs. [7, [10] and extend the electroweak model to include an extra $U(1)$. So we consider the $S U(2)_{L} \times U(1)_{Y} \times U(1)_{F}$ invariant lagrangian

$$
\mathcal{L}=-\frac{1}{4} G_{\mu \nu}^{a} G^{\mu \nu a}-\frac{1}{4} B_{\mu \nu} B^{\mu \nu}-\left(D_{\mu} h\right)^{\dagger}\left(D^{\mu} h\right)-\frac{1}{4} X_{\mu \nu} X^{\mu \nu}-\left(D_{\mu} \phi\right)^{*}\left(D^{\mu} \phi\right)-V(h, \phi)
$$

where the potential is given by

$$
V(h, \phi)=\lambda_{h}\left(|h|^{2}-\frac{v_{h}^{2}}{2}\right)^{2}+\lambda_{\phi}\left(|\phi|^{2}-\frac{v_{\phi}^{2}}{2}\right)^{2}+f\left(|h|^{2}-\frac{v_{h}^{2}}{2}\right)\left(|\phi|^{2}-\frac{v_{\phi}^{2}}{2}\right)
$$


and the covariant derivatives are

$$
\begin{aligned}
D_{\mu} \phi & =\left(\partial_{\mu}-i \frac{q}{2} F_{\phi} X_{\mu}\right) \phi \\
D_{\mu} h & =\left(\partial_{\mu}-i \frac{g}{2} \sigma^{a} W_{\mu}^{a}-i \frac{g^{\prime}}{2} B_{\mu}-i \frac{q}{2} F_{h} X_{\mu}\right) h
\end{aligned}
$$

where $\sigma^{a}$ are the Pauli spin matrices, $W_{\mu}^{a}$ are the $S U(2)_{L}$ gauge fields, $B_{\mu}$ are the $U(1)_{Y}$ gauge fields and $X_{\mu}$ are the $U(1)_{F}$ gauge fields. $h$ is the electroweak Higgs doublet and has a coupling to the $U(1)_{F}$ gauge field, while $\phi$ is an electroweak singlet.

If we assume that this symmetry group is unified into a simple Lie group $G$ then the $U(1)_{F}$ charges $F_{\phi}$ and $F_{h}$ will in general be rational, but we cannot characterize them further without specifying $G$.

The $U(1)_{F}$ symmetry is broken first and gives rise to topologically stable string solutions of the form

$$
\phi=\phi_{0} S(r) e^{i N \theta}, \quad X_{\theta}=\frac{2 N}{q F_{\phi}} \frac{A(r)}{r},
$$

where $S(r)$ and $A(r)$ are Nielsen-Olesen profiles [11], and $N$ is the winding number. The string is taken to be along the $\mathrm{z}$ axis. The scalar field $\phi$ shall be taken as a GUT scale field and so $\phi_{0} \simeq 10^{16} \mathrm{GeV}$, whereas the Higgs field acquires a vev of the order $10^{2} \mathrm{GeV}$. Since the characteristic scale over which a field of mass $m$ varies is of the order $1 / m$, we see that the characteristic scale of $h$ is fourteen orders of magnitude bigger than that of $\phi$. So the internal structure of the GUT string is irrelevant and we need only consider the asymptotic forms of the Nielsen-Olesen string which are $S(r)=1$ and $A(r)=1$ for $r \rightarrow \infty$.

We first consider the case $F_{h}=0$, when the potential term is the only coupling between the Higgs doublet and the GUT string. The minimum of the potential is given by

$$
\begin{aligned}
|h|=0 & \text { or } & |h|^{2}=\frac{v_{h}^{2}}{2}-\frac{f}{2 \lambda_{h}}\left(|\phi|^{2}-\frac{v_{\phi}^{2}}{2}\right), \\
|\phi|=0 & \text { or } & |\phi|^{2}=\frac{v_{\phi}^{2}}{2}-\frac{f}{2 \lambda_{\phi}}\left(|h|^{2}-\frac{v_{h}^{2}}{2}\right) .
\end{aligned}
$$

The vacuum values are given by $|\phi|^{2}=v_{\phi}^{2} / 2$ and $|h|^{2}=v_{h}^{2} / 2$ with $v_{\phi}^{2} \gg v_{h}^{2}$. If we consider a region where $|\phi|=0$ then the potential energy is minimized by

$$
|h|^{2}=\frac{v_{h}^{2}}{2}+\frac{f v_{\phi}^{2}}{4 \lambda_{h}} .
$$

We can see that for $f>0$ the expectation value of the Higgs is likely to be raised in the string core, while for $f<0$ it is lowered. For $f$ sufficiently negative $|h|^{2}$ can become less than zero so we must take $|h|=0$. Consequently the electroweak symmetry can be restored about a GUT string; this is the result given in [10]. Note that electroweak 
symmetry restoration for $F_{h}=0$ only occurs for a range of parameters, and that the above considerations do not give this range because we have ignored the self-energy potential terms and the kinetic terms. Conversely, for $f>0$ the electroweak symmetry is always broken in a region of size $m_{h}^{-1}$ arround the GUT string [12].

We now look for a solution of the form $h^{\dagger}=\left(0, h_{d}^{*}\right)$ in the background of the GUT string. Since the GUT string is so massive the back-reaction of an electroweak field configuration on the GUT string will be negligible. The equation of motion for $h_{d}$ is

$$
-\nabla^{2} h_{d}+2 \lambda_{h}\left(\left|h_{d}\right|^{2}-\frac{v_{h}^{2}}{2}\right) h_{d}+f\left(|\phi|^{2}-\frac{v_{\phi}^{2}}{2}\right) h_{d}=0 .
$$

Now for the GUT string $\phi=v_{\phi} S(r) \exp (i \theta) / \sqrt{2}$, where $S(r)$ is a Nielsen-Olesen profile, and so

$$
f\left(|\phi|^{2}-\frac{v_{\phi}^{2}}{2}\right)=\frac{f v_{\phi}^{2}}{2}\left(S^{2}-1\right) .
$$

The width of the GUT string is approximately $1 / \sqrt{\lambda_{\phi} v_{\phi}^{2}}$ and so for $v_{\phi} \gg v_{h}$ the potential cross term is well approximated by a delta function $\delta(r)$. For $f$ large and negative the delta function gives the boundary condition at the origin (taken to be the location of the GUT string), $h_{d}^{\prime}(0)=0$. The profile obtained by solving the equation of motion for $h_{d}$ with this boundary conditions is shown in Figure 1. Note that it does not appear to satisfy $h_{d}^{\prime}(0)=0$. This is because on the GUT scale the Higgs gradient is given by $h_{d}^{\prime}\left(r_{\phi}\right)=f m_{\phi} h_{d}\left(r_{\phi}\right) / 2 \lambda_{\phi}$, and we are considering the limit when $r_{\phi}=1 / m_{\phi} \rightarrow 0$.

Essentially, the electroweak symmetry is restored completely on the scale for which $|\phi|^{2}=0$, i.e. on the GUT scale, then $h_{d}$ returns to its vacuum value over its characteristic length scale $1 / m_{h}$.

Now when $F_{h} \neq 0$ the potential term is irrelevant, except possibly for very large positive values of the parameter $f$. This is because the energy density has a contribution of the form $\left|X_{\theta} h\right|^{2}$ from the covariant derivative, which for the GUT gauge field $X_{\theta}=$ $2 N / q F_{\phi} r$ and the vacuum $h_{d}=v_{h} / \sqrt{2}$, will give a logarithmically divergent contribution to the energy per unit length [10, 13].

To cancel this logarithmic part of the $\theta$ covariant derivative

$$
D_{\theta} h_{d}=\left(\frac{1}{r} \frac{\partial}{\partial \theta}+\frac{i g_{z}}{2} Z_{\theta}-\frac{i F_{h}}{F_{\phi}} \frac{N}{r}\right) h_{d}
$$

requires either a $\theta$ dependence for $h_{d}, Z_{\theta} \neq 0$ or both. So consider the field configuration

$$
h_{d}(r, \theta)=h_{d}(r) \exp \left(i \alpha \frac{F_{h}}{F_{\phi}} \theta\right), \quad Z_{\theta}=\frac{\gamma F_{h}}{F_{\phi}} \frac{2 a(r)}{g_{z} r},
$$

where we take $a(r) \rightarrow 1$ as $r \rightarrow \infty$, and $\alpha$ such that $\alpha F_{h} / F_{\phi}$ is an integer, so that $h_{d}$ is a single valued function of $\theta$. Substituting these fields into the covariant derivative above gives

$$
D_{\theta} h_{d}=\frac{i F_{h}}{F_{\phi}} \frac{h_{d}(r, \theta)}{r}(\alpha+\gamma a(r)-N)
$$


and so to cancel the logarithmic divergence requires $\alpha+\gamma=N$. Using this we can rewrite the covariant derivative as

$$
D_{\theta} h_{d}=\frac{i \gamma F_{h}}{F_{\phi}} \frac{h_{d}(r, \theta)}{r}(a(r)-1)
$$

Then the energy of the above configuration is given by

$$
E=\pi v_{h}^{2} \int r d r\left[\left(\frac{d h_{d}}{d r}\right)^{2}+\left(\frac{\gamma F_{h}}{F_{\phi}}\right)^{2} \frac{(a-1)^{2}}{r^{2}} h_{d}^{2}+\frac{\beta}{2}\left(h^{2}-1\right)^{2}+\left(\frac{\gamma F_{h}}{F_{\phi}}\right)^{2} \frac{1}{2 r^{2}}\left(\frac{d a}{d r}\right)^{2}\right]
$$

where we have rescaled $h_{d} \rightarrow h_{d} v_{h} / \sqrt{2}, Z_{\theta} \rightarrow Z_{\theta} v_{h} / \sqrt{2}, r \rightarrow r 2 \sqrt{2} / g_{z} v_{h}$ and $\beta=8 \lambda_{h} / g_{z}^{2}$, with $g_{z}^{2}=g^{2}+g^{\prime 2}$. We are using the standard field basis of $W_{\mu}^{+}, W_{\mu}^{-}, Z_{\mu}$ and $A_{\mu}$ for the electroweak fields. This expression for the energy is the same as for the NielsenOlesen string but with the winding number replaced by $-\gamma F_{h} / F_{\phi}$, which is in general non-integer. The profiles $h_{d}(r)$ and $a(r)$ will therefore be string-like, as can be seen in Figure 2, and the energy per unit length in the electroweak fields is $\sim \pi v_{h}^{2}$.

Electroweak strings are non-topological and it is possible for them to unwind via 'W-condensation' [14] to the electroweak vacuum. In the case we are considering it is not possible for the string-like solution to decay to the electroweak vacuum, because of the logarithmic term in the energy that would result. There are, however, a range of possible values of $\alpha$ and $\gamma$ that satisfy the condition $\alpha+\gamma=N$. To see which values give stable string-like solutions, we consider $h_{u}$ and $W_{\mu}^{+}$perturbations about the solution and look for negative modes. Since the GUT string fields are so massive we need not consider perturbations in the GUT string fields. This means that the perturbations about the string-like solution above, give rise to the same perturbation equations as in the electroweak string case [15] but with the winding number replaced by $-\gamma F_{h} / F_{\phi}$. We expand the perturbations as

$$
\delta h_{u}=\sum_{m^{\prime}} s_{m}^{\prime}(r) e^{i m^{\prime} \theta} e^{i \omega t}, \quad \delta W_{\uparrow}^{+}=\sum_{m}-i w_{m} e^{i(m-1) \theta} e^{i \omega t}, \quad \delta W_{\downarrow}^{+}=\sum_{m} i w_{m}^{*} e^{i(m+1) \theta} e^{i \omega t}
$$

where $m^{\prime}=m+\left(\alpha F_{h} / F_{\phi}\right)$. The symbols $\uparrow$ and $\downarrow$ refer to the component of spin along the $\mathrm{z}$ axis being +1 or -1 respectively. The resulting perturbation equations in the background gauge

$$
\partial^{\mu} \delta W_{\mu}^{+}-i g \cos \theta_{W} Z^{\mu} \delta W_{\mu}^{+}-\frac{i g}{\sqrt{2}} h_{d}^{*} \delta h_{u}=0
$$

are

$$
\left(\begin{array}{ccc}
D_{1} & A & B \\
A & D_{2} & 0 \\
B & 0 & D_{3}
\end{array}\right)\left(\begin{array}{c}
s_{m^{\prime}} \\
w_{m} \\
w_{-m}^{*}
\end{array}\right)=\omega^{2}\left(\begin{array}{c}
s_{m^{\prime}} \\
w_{m} \\
w_{-m}^{*}
\end{array}\right)
$$

where

$$
D_{1}=-\nabla_{\rho}^{2}+\frac{\left(m^{\prime}-a \epsilon \cos 2 \theta_{W}\right)^{2}}{\rho^{2}}+\beta\left(f^{2}-1\right)+2 f^{2} \cos ^{2} \theta_{W}
$$




$$
\begin{aligned}
D_{2} & =-\nabla_{\rho}^{2}+\frac{\left((m-1)-2 a \epsilon \cos ^{2} \theta_{W}\right)^{2}}{\rho^{2}}+2 f^{2} \cos ^{2} \theta_{W}-4 \cos ^{2} \theta_{W} \frac{\epsilon}{\rho} \frac{d a}{d \rho} \\
D_{3} & =-\nabla_{\rho}^{2}+\frac{\left((m+1)-2 a \epsilon \cos ^{2} \theta_{W}\right)^{2}}{\rho^{2}}+2 f^{2} \cos ^{2} \theta_{W}+4 \cos ^{2} \theta_{W} \frac{\epsilon}{\rho} \frac{d a}{d \rho} \\
A & =2 \cos \theta_{W}\left(\nabla_{\rho} f+\frac{\epsilon f}{\rho}(1-a)\right) \\
B & =-2 \cos \theta_{W}\left(\nabla_{\rho} f-\frac{\epsilon f}{\rho}(1-a)\right)
\end{aligned}
$$

and we have rescaled as before. The parameter $\epsilon$ is $\gamma F_{h} / F_{\phi}$. There are two terms in the perturbation equations above which can give negative contributions; they are the potential term in $D_{1}$ and the last term of $D_{2}$. This latter term corresponds to a $-\mathbf{m}$.B interaction energy between the Z-magnetic moment (m) of the W-boson and the Zmagnetic field $(\mathbf{B})$ of the string-like solution.

We know that for integer values of $\epsilon$ (which will occur for $F_{h} / F_{\phi}$ an integer ) the string solution has negative eigenvalues for modes corresponding to the W-bosons acquiring nonzero values in the core of the string [15, [16]. But if the equations of motion are solved with this 'W-condensate', it has been shown that the solution is gauge equivalent to a string of lower winding number [14]. In the case of the string-like solution the equations of motion for a 'W-condensate' are the same as in ref. [14] but with the winding number replaced by $-\epsilon$. Since the generator of the GUT string acts on the electroweak doublet as a constant times the identity, and the GUT scalar field is an electroweak singlet, the presence of the GUT string does not prevent a similar gauge transformation from being made. This will still be true for non-integer values of $\epsilon$. So if we find negative modes to the equations above, we must distinguish between those which are 'W-condensation' and those which result in a physical W-boson condensate trapped in the string core. The former are unwindings of the string while the later would give a charged condensate which would break the $U(1)$ of electromagnetism and so give rise to superconductivity.

If we consider the energy expression (1) we see that the energy is lower for smaller values of $\left|\gamma F_{h} / F_{\phi}\right|$. If we consider

$$
\frac{\alpha F_{h}}{F_{\phi}}+\frac{\gamma F_{h}}{F_{\phi}}=\frac{N F_{h}}{F_{\phi}},
$$

then since $N F_{h} / F_{\phi}$ is fixed and $\alpha F_{h} / F_{\phi}$ can only change by an integer, we conclude that $\gamma F_{h} / F_{\phi}$ (the $Z$-flux of the string-like solution in units $4 \pi / g_{z}$ ) can only change by an integer. We would expect this lowering of the flux by integer amounts to occur by 'W-condensation' for all $\epsilon$, as it does for integer $\epsilon$. Thus we expect $\epsilon$ to be lowered by integer amounts until it lies in the range $-\frac{1}{2}<\epsilon<\frac{1}{2}$. If the string-like solutions with $\epsilon$ in this range were to have any negative modes they could not be interpreted as unwindings since they would raise the energy, and so would have to be interpreted as the occurrence of a physical charged condensate. 
To investigate the above arguments numerically for a GUT string of winding $N=1$, we consider $F_{h} / F_{\phi}$ values of (a) 0 , (b) 1 , (c) 0.4 and (d) 0.5 . Case (c) is actually realised in an $S O(10)$ model considered by Alford and Wilczek [17].

For case (a) the $\delta$-function potential cross term is the only coupling between the GUT string and the electroweak fields, and its only effect is to give $h_{d}(0)^{\prime}=0$. As seen earlier, this condition is satisfied on the GUT scale but is negligible on the electroweak scale, and so for string solutions the effect of the potential term on the profiles is negligible. So the string solutions and their stability are the same as for electroweak strings solutions; they are unstable for physical values of parameters [15, 16]. The 'vacuum' solution in this case is that shown in Figure 1.

For case (b) $\epsilon$ will be an integer, and so the string solutions and their stability will again be the same as for the electroweak string. The 'vacuum' in this case will have $\epsilon=0$, but the Higgs field will still have a winding in order to cancel the logarithmic contribution to the energy from the GUT gauge field. The 'vacuum' solution is again given by the profile in Figure 1.

For case (c) first consider $(\alpha, \gamma)=(5 / 2,-3 / 2)$ which gives $\epsilon=-0.6$ and is outside our proposed stability range. The profiles for the string-like configuration were solved for by a relaxation method on the energy (1) and substituted into the perturbation equations. These were then solved by direct matrix methods for $\sin ^{2} \theta_{w}=0.23$. A negative mode was found for angular momentum $m=-1$. As with the electroweak string, this mode is interpreted as an instability to the winding $(\epsilon)$ increasing by one unit. The stability line i.e. the line in $\left(\beta, \theta_{w}\right)$ parameter space for which $\omega^{2}=0$, is an approximate vertical line at about $\theta_{w}=\pi / 4$. For $\epsilon<-0.6$ this line moves up to higher $\theta_{w}$, while for $\epsilon>-0.6$ this line moves down to lower $\theta_{w}$ and so for some $\epsilon$ we would expect no negative modes to occur at $\sin ^{2} \theta_{w}=0.23$.

Now consider case (c) with $(\alpha, \gamma)=(0,1)$ which gives $\epsilon=0.4$, i.e. it is the solution the above configuration decayed to. This had no negative modes and so is a stable solution.

For case (d) the parameter values $(\alpha, \gamma)=(0,1)$ and $(\alpha, \gamma)=(2,-1)$ have $\epsilon$ values of +0.5 and -0.5 respectively and so the two solutions are degenerate in energy. For $\sin ^{2} \theta_{w}=0.23$ both of these solutions were found to be stable. For $\sin ^{2} \theta_{w}=0$ the $\epsilon=-0.5$ solution was found to have an $m=-1$ zero mode while the $\epsilon=0.5$ solution had as $m=1$ zero mode. Integer $\epsilon$ strings also have zero modes at $\sin ^{2} \theta_{w}=0$, and these also occur at angular momentum $m=2 \epsilon$ [15]. These modes are to be interpreted as transitions between the $-\epsilon$ and $+\epsilon$ solutions via a W-string. For $\sin ^{2} \theta_{w}>0$ the energy of the $\mathrm{W}$-string is above that of the corresponding Z-string and so there is a barrier to such transitions, while for $\sin ^{2} \theta_{w}=0$ the $\mathrm{W}$-string and Z-string solutions are degenerate in energy.

So at $\sin ^{2} \theta_{w}=0$ all strings with $|\epsilon| \leq 0.5$ are stable. At $\sin ^{2} \theta_{w}=0.23$, in addition to the stable strings above there are metastable string solutions for $|\epsilon|$ in the approximate range $0.51-0.53$ for $\beta$ in the range $0.25-4.0$.

Now in [7] it was claimed that because the electroweak symmetry was restored the $\mathrm{W}$-bosons would be massless, since the $\mathrm{W}$-boson gets its mass from a term proportional 
to $\left|h_{d}\right|^{2}$. The confinement energy was, however, ignored and since the potential well is approximately $1 / m_{h}$ wide and the characteristic scale of the $\mathrm{W}$-boson $1 / m_{W}$ is comparable, the confinement energy will be sizeable.

So we looked at the W-boson bound modes at $\sin ^{2}\left(\theta_{w}\right)=0.23$ for $\sqrt{\beta}=0.5,1.0$ and 2.0 for the various cases above. The eigenvalues obtained for the bound $\mathrm{W}$-bosons in case (a) with angular momentum $m=0$, are $\omega=0.88,0.91$ and $0.92 m_{W}$ respectively $\left(m_{W}\right.$ is the mass of the $\mathrm{W}$-boson in the vacuum), which in view of the above comments is to be expected. The remaining cases also possessed bound W-bosons for angular momentum $m=0$ with similar sized eigenvalues.

For angular momentum $m=-1$ the most likely case to have bound W-bosons with significantly lower eigenvalues than above is $|\epsilon|=0.5$, since there are zero modes at $\sin ^{2} \theta_{w}=0$. We found that for $\epsilon=0.5$ the lowest bound mode eigenvalues are $\omega=0.25$, 0.27 and $0.28 m_{W}$ respectively. These are the lowest bound mode eigenvalues that were obtained for any of the stable strings for the $\theta_{w}$ and $\beta$ values given above. We therefore find no massless $\mathrm{W}$-boson states on the strings [19].

Finally we consider whether there are any fermion zero modes present on the stable string-like configurations. We know that electroweak strings possess fermion zero modes [20] and so we might expect there to be fermion zero modes on the string-like solutions as well. To investigate the possible existence of fermion zero modes consider the $S U(2)_{L} \times$ $U(1)_{Y} \times U(1)_{F}$ invariant lagrangian for the first family of leptons

$$
\mathcal{L}_{\text {ferm }}=-i \bar{\psi} \gamma^{\mu} D_{\mu} \psi-i e_{R} \gamma^{\mu} D_{\mu} e_{R}+Y_{e}\left(e_{R} h^{\dagger} \psi+\bar{\psi} h e_{R}\right)
$$

where

$$
\begin{aligned}
D_{\mu} \psi & =\left(\partial_{\mu}-\frac{i g \tau^{a}}{2} W_{\mu}^{a}+\frac{i g^{\prime}}{2} B_{\mu}-\frac{i q}{2} F_{L}^{e} X_{\mu}\right) \psi \\
D_{\mu} e_{R} & =\left(\partial_{\mu}+i g^{\prime} B_{\mu}-\frac{i q}{2} F_{R}^{e} X_{\mu}\right) e_{R}
\end{aligned}
$$

and $Y_{e}$ is a constant. For the Yukawa coupling term to be $U(1)_{F}$ invariant we must have $F_{L}^{e}-F_{R}^{e}=F_{h}$.

The Dirac equations in the presence of the GUT string with an electroweak string-like configuration about it are

$$
\begin{aligned}
& \left(i D_{0}+i \sigma_{k} D_{k}\right) e_{L}=m h_{d} e_{R} \\
& \left(i D_{0}-i \sigma_{k} D_{k}\right) e_{L}=m h_{d}^{*} e_{R}
\end{aligned}
$$

where

$$
\begin{aligned}
D_{k} e_{L} & =\left(\partial_{k}+\frac{i g_{z}}{2} \cos 2 \theta_{w} Z_{k}-\frac{i q}{2} F_{L}^{e} X_{k}\right) e_{L} \\
D_{k} e_{R} & =\left(\partial_{k}-i g_{z} \sin ^{2} \theta_{w} Z_{k}-\frac{i q}{2} F_{R}^{e} X_{k}\right) e_{R}
\end{aligned}
$$


Now we write $e_{R}^{T}=\left(c_{1}, c_{2}\right), e_{L}^{T}=\left(d_{1}, d_{2}\right)$ and expand $c_{1}, c_{2}, d_{1}$ and $d_{2}$ as

$$
\begin{aligned}
c_{1} & =c_{1}(r) \exp \left(i k z-i \omega t+i\left(m-\frac{\alpha F_{h}}{F_{\phi}}\right) \theta\right) \\
c_{2} & =i c_{2}(r) \exp \left(i k z-i \omega t+i\left(m+1-\frac{\alpha F_{h}}{F_{\phi}}\right) \theta\right) \\
d_{1} & =d_{1}(r) \exp (i k z-i \omega t+i m \theta) \\
d_{2} & =i d_{2}(r) \exp (i k z-i \omega t+i(m+1) \theta)
\end{aligned}
$$

where $m$ is the angular momentum of the mode. We are looking for zero modes so we consider $|\omega|=|k|=0$. Then the equations separate into two pairs of coupled equations,

$$
\begin{aligned}
d_{1}^{\prime}-\frac{d_{1}}{r}\left[\left(m-\frac{N F_{L}^{e}}{F_{\phi}}\right)+\frac{\gamma F_{h}}{F_{\phi}} a(r) \cos 2 \theta_{w}\right] & =Y_{e} h_{d}(r) c_{2} \\
c_{2}^{\prime}+\frac{c_{2}}{r}\left[\left(m+1-\frac{\alpha F_{h}}{F_{\phi}}-\frac{N F_{R}^{e}}{F_{\phi}}\right)-\frac{\gamma F_{h}}{F_{\phi}} a(r) 2 \sin ^{2} \theta_{w}\right] & =Y_{e} h_{d}(r) d_{1}
\end{aligned}
$$

and

$$
\begin{aligned}
c_{1}^{\prime}-\frac{c_{1}}{r}\left[\left(m-\frac{\alpha F_{h}}{F_{\phi}}-\frac{N F_{R}^{e}}{F_{\phi}}\right)-\frac{\gamma F_{h}}{F_{\phi}} a(r) 2 \sin ^{2} \theta_{w}\right] & =-Y_{e} h_{d}(r) d_{1} \\
d_{2}^{\prime}+\frac{d_{2}}{r}\left[\left(m+1-\frac{N F_{L}^{e}}{F_{\phi}}\right)+\frac{\gamma F_{h}}{F_{\phi}} a(r) \cos 2 \theta_{w}\right] & =-Y_{e} h_{d}(r) c_{1}
\end{aligned}
$$

For there to be a zero mode solution both fields in the pair must be non-singular at the origin [21]. For (2) this requires

$$
m-\frac{N F_{L}^{e}}{F_{\phi}} \geq 0 \text { and } \quad \frac{\alpha F_{h}}{F_{\phi}}+\frac{N F_{R}^{e}}{F_{\phi}}-m-1 \geq 0 .
$$

Using $F_{R}^{e}=F_{L}^{e}-F_{h}$ gives

$$
m \geq \frac{N F_{L}^{e}}{F_{\phi}} \quad \text { and } \quad(\alpha-N) \frac{F_{h}}{F_{\phi}}+\frac{N F_{L}^{e}}{F_{\phi}}-1 \geq m
$$

and so for both conditions to be true, we must have

$$
\gamma \frac{F_{h}}{F_{\phi}} \leq-1
$$

where we used $\alpha+\gamma=N$. Similarly for (3) we get

$$
\gamma \frac{F_{h}}{F_{\phi}} \geq 1
$$


So for there to be fermion zero modes we must have $\left|\gamma F_{h} / F_{\phi}\right| \geq 1$ but we showed earlier that the string-like solutions are only stable for $\left|\gamma F_{h} / F_{\phi}\right| \leq 1 / 2$, and so the stable solutions do not have fermion zero modes.

So in conclusion we find that if the only coupling between the GUT string fields $\left(\phi, X_{\theta}\right)$ and the electroweak fields is a $q F_{h} X_{\mu} h / 2$ term in the covariant derivative and a $|\phi|^{2}|h|^{2}$ term in the potential, then there are electroweak string-like solutions about the GUT string. The Z-flux of such strings is $\Phi_{Z}=\left(n-F_{h} N / F_{\phi}\right) 4 \pi \cos \theta_{w} / g$, where $n$ is an integer and $N$ is the integer winding of the GUT string. We found no evidence for the formation of stable charged condensates: the strings with $\left|n-F_{h} N / F_{\phi}\right|>1 / 2$ did possess negative modes, but we surmise these instabilities are due to the string decaying to one with lower Z-flux by the 'W-condensation' mechanism of ref. [14. Those strings with $\left|n-F_{h} N / F_{\phi}\right| \leq 1 / 2$ possess no negative modes and so GUT strings can have stable electroweak strings arround them, similar to those found arround global strings in a two Higgs doublet model in ref. [22].

The mechanism for superconductivity given in ref. [7] required the occurrence of Wboson zero modes on the string. We have shown that these do not occur for this class of string solutions and so supercurrents do not arise as claimed in refs. [7, 8].

We have further shown that the stable string solutions do not possess fermion zero modes, and so conclude that a non-superconducting GUT string does not become superconducting after the electroweak phase transition.

The effects of there being stable electroweak string solutions about GUT strings should be negligible. First of all, particle production by the string due to the coupling between the GUT string and light particles has been considered in ref. [23], where it was shown that gravitational radiation was a more significant energy loss mechanism. We would not expect any significant change in the dynamics of the GUT strings due to the forces between the electroweak strings because they are negligible in comparison to the GUT string mass.

In ref. [24] a baryon production mechanism was outlined which involved the de-linking of linked electroweak strings. Electroweak strings are, however, highly unstable and so it is unclear whether or not they form. Here we have stable electroweak strings forming about GUT strings. However, the GUT string network will have reached a scaling solution by the electroweak phase transition and so the number density of linked strings would be extremely low. The net baryon number produced by this mechanism would be negligible.

\section{Acknowledgements}

This work was supported by PPARC: MG by studentship number 93300941 and MH by Advanced Fellowship number B/93/AF/1642.

\section{References}


[1] A. Vilenkin and E.P.S. Shellard, Cosmic Strings and Other Topological Defects (Cambridge University Press, Cambridge, 1994)

[2] M.B. Hindmarsh and T.W.B. Kibble, Rep. Prog. Phys. 58, 477 (1995)

[3] R. Jeannerot and A. C. Davis, hep-ph/9501275

[4] E. Witten, Nucl. Phys. B249, 557 (1985)

[5] A. E. Everett, Phys. Rev. Lett. 61, 1807 (1988)

[6] M. Alford, K. Benson, S. Coleman and J. March Russel, Nucl. Phys. B349, 439 (1991)

[7] P. Peter, Phys. Rev. D49, 5052 (1994)

[8] A. C. Davis and P. Peter, hep-ph/9506433

[9] J. Ambjørn, N. K. Nielsen and P. Olesen, Nucl. Phys. B310, 625 (1988)

[10] W. B. Perkins and A. C. Davis, Nucl. Phys. B406, 377 (1993)

[11] H. Nielsen and P. Olesen, Nucl. Phys. B61, 45 (1973)

[12] U. A. Yajnik, Phys. Rev. D34, 1237 (1986)

[13] M. Hindmarsh, Phys. Lett. B225, 127 (1989)

[14] A. Achúcarro, R. Gregory, J. A. Harvey and K. Kuijken, Phys. Rev. Lett. 72, 3646 (1994)

[15] M. Goodband and M. Hindmarsh, hep-ph/9505357 (to be published in Phys. Lett. B)

[16] M. James, L. Perivolaropoulos and T. Vachspati, Nucl. Phys. B395, 534 (1993)

[17] M. G. Alford and F. Wilczek, Phys. Rev. Lett. 62, 1071 (1989)

[18] M. Barriola, T. Vachaspati and M. Bucher, Phys. Rev. D50, 2819 (1994)

[19] The author of ref. 7 is now in agreement on this point (P. Peter, private communication, 1995)

[20] M. A. Earnshaw and W. B. Perkins, hep-ph/9402218

[21] R. Jackiw and P. Rossi, Nucl. Phys. B190, 681 (1981)

[22] G. Dvali and G. Senjanovic, Phys. Rev. Lett. 71, 2376 (1993)

[23] M. Srednicki and S. Theisen, Phys. Lett. B189, 397 (1987)

[24] T. Vachaspati, Phys. Rev. Lett. 73, 373 (1994) 


\section{Figure captions}

Figure 1: $h_{d}$ profile showing symmetry restoration about a GUT string for $F_{h}=0$.

Figure 2: $h_{d}(r)$ and $a(r)$ profiles for $\epsilon=0.4$ (solid line) and those for a Nielsen-Olesen string (dashed line) ( $\epsilon=1$ for comparison) 
Figure 1.

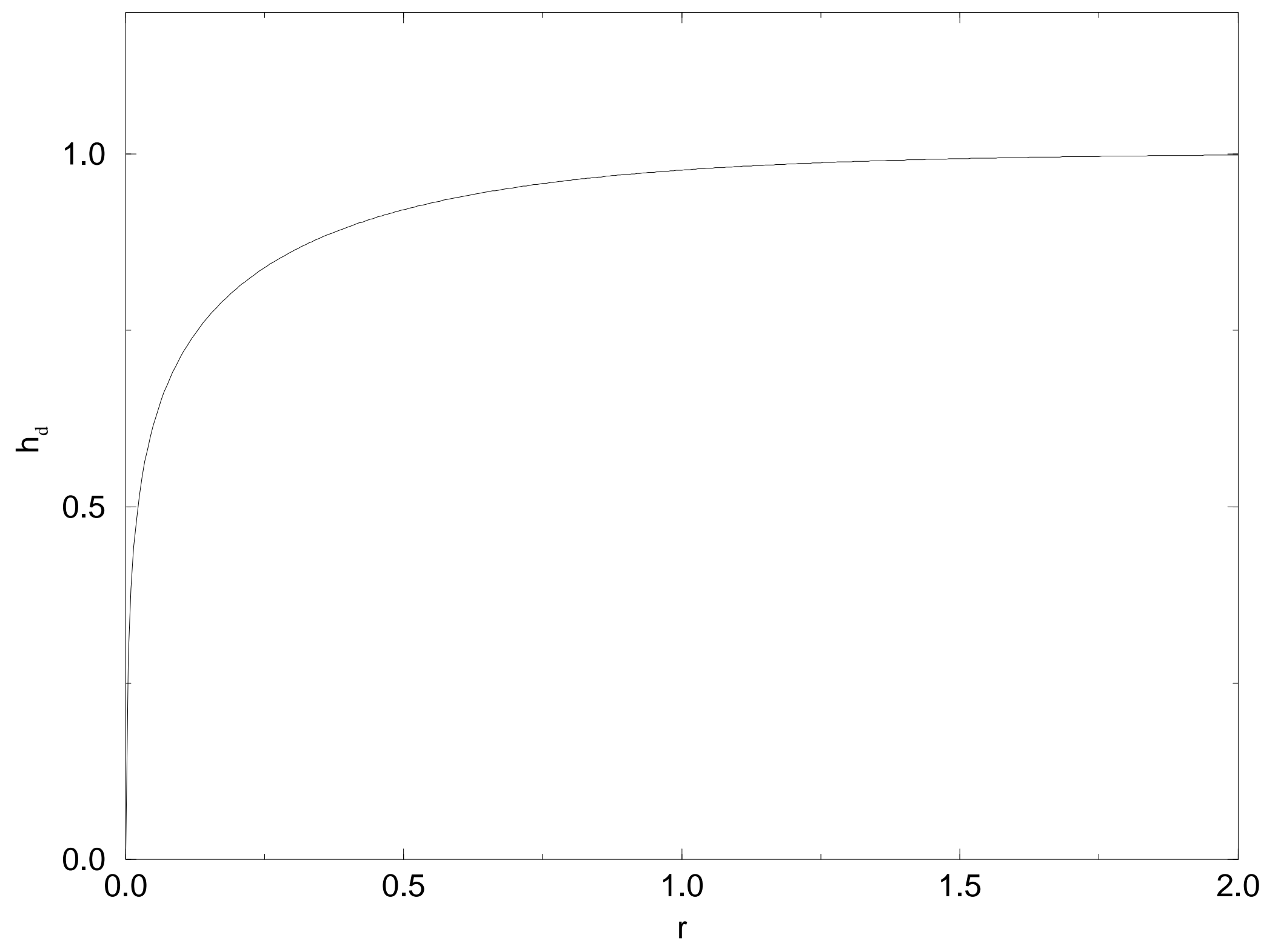


Figure 2.

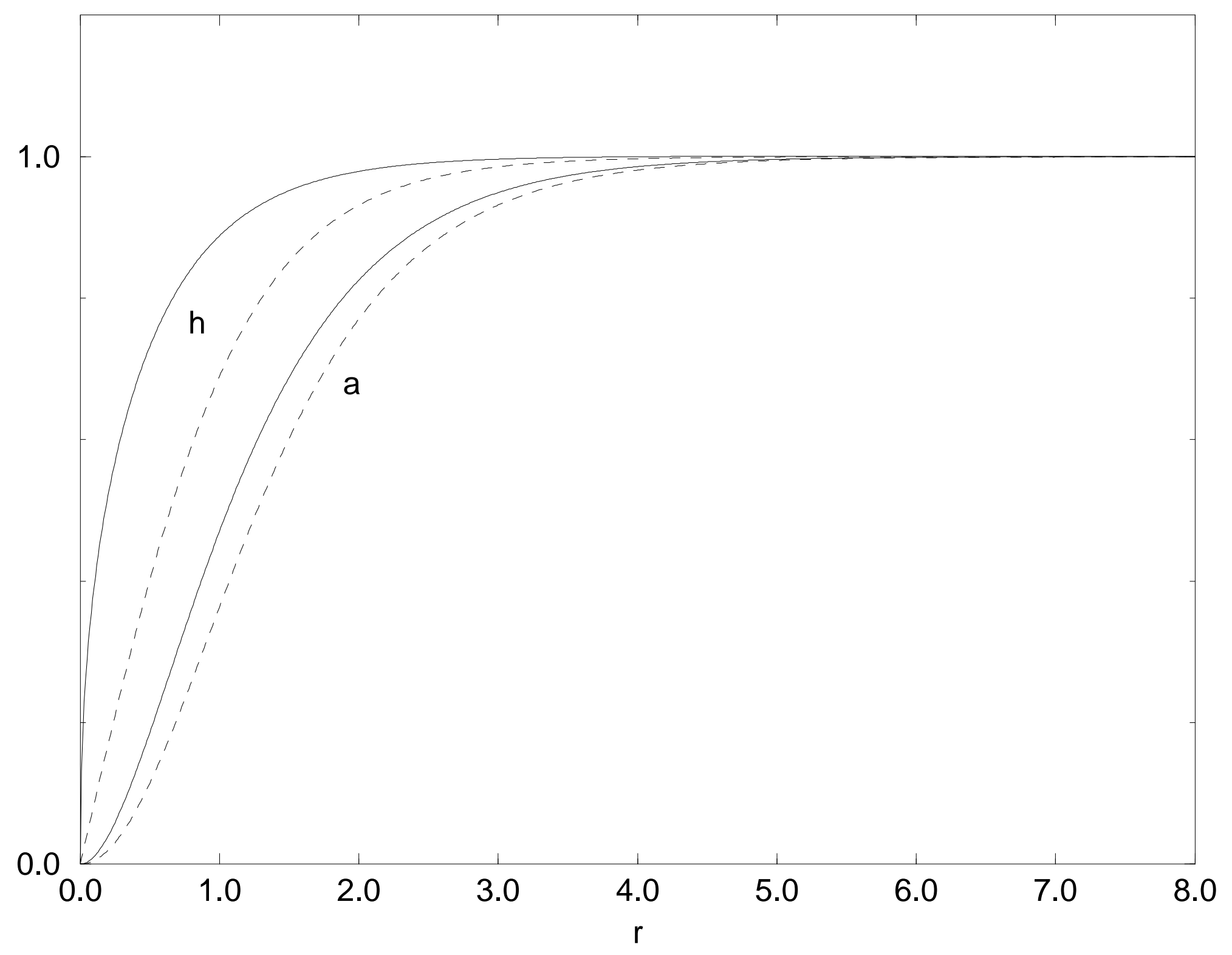

